\title{
Quantifying direct DNA damage in the basal layer of skin exposed to UV radiation from sunbeds
}

\begin{tabular}{|r|l|}
\hline Journal: & Photochemistry and Photobiology \\
\hline Manuscript ID & PHP-2018-01-RA-0007.R1 \\
\hline Wiley - Manuscript type: & Research Article \\
\hline Date Submitted by the Author: & 31 -Mar-2018 \\
\hline Complete List of Authors: & $\begin{array}{l}\text { Barnard, Isla; University of St Andrews, School of Physics and Astronomy } \\
\text { Tierney, Patrick; Ninewells Hospital, Photobiology Unit } \\
\text { Campbell, Catherine Louise; University of St. Andrews, School of Physics \& } \\
\text { Astronomy } \\
\text { McMillan, Lewis; University of St. Andrews, School of Physics \& Astronomy } \\
\text { Moseley, Harry } \\
\text { Eadie, Ewan; Ninewells Hospital, Photobiology Unit } \\
\text { Brown, C; University of St. Andrews, School of Physics \& Astronomy } \\
\text { Wood, Kenny; University of St. Andrews, School of Physics and Astronomy }\end{array}$ \\
\hline Keywords: & \begin{tabular}{l} 
UV, UVA, DNA Damage \\
\hline
\end{tabular} \\
\hline
\end{tabular}


1 Quantifying direct DNA damage in the basal layer of skin exposed

\section{to UV radiation from sunbeds}

3 Isla Rose Mary Barnard ${ }^{\mathbf{1}}$, Patrick Tierney ${ }^{2}$, Catherine Louise Campbell ${ }^{2}$, Lewis

4 McMillan ${ }^{1}$, Harry Moseley ${ }^{2}$, Ewan Eadie ${ }^{2}$, Christian Tom Alcuin Brown ${ }^{1}$, Kenneth

Wood $^{1}$

6

$10{ }^{1}$. SUPA, School of Physics and Astronomy, University of St Andrews, North Haugh, St

11 Andrews, KY16 9SS, UK, ${ }^{2}$. Photobiology Unit, Ninewells Hospital \& Medical School,

Dundee, DD1 9SY, UK 


\section{ABSTRACT}

16 Non-melanoma and melanoma skin cancers are attributable to DNA damage caused by 17 ultraviolet (UV) radiation exposure. One DNA photoproduct, the Cyclobutane Pyrimidine

18 Dimer (CPD), is believed to lead to DNA mutations caused by UV radiation. Using radiative

19 transfer simulations, we compare the number of CPDs directly induced by UV irradiation from

20 artificial and natural UV sources (a standard sunbed and the midday summer Mediterranean

21 sun) for skin types I and II on the Fitzpatrick scale. We use Monte Carlo Radiative Transfer

22 (MCRT) modelling to track the progression of UV photons through a multi-layered three

23 dimensional (3D) grid that simulates the upper layers of the skin. By recording the energy

24 deposited in the DNA containing cells of the basal layer, the number of CPDs formed can be

25 quantified. The aim of this work was to compare the number of CPDs formed in the basal

26 layer of the skin, and by implication the risk of developing cancer, as a consequence of

27 irradiation by artificial and natural sources. Our simulations show that the number of CPDs

28 formed per second during sunbed irradiation is almost three times that formed during solar 29 irradiation. 


\section{INTRODUCTION}

32 Over-exposure to ultraviolet (UV) radiation is a major cause of skin cancer $(1,2)$ although the

33 risks from solar UV radiation can be reduced by covering skin, or by using sunscreen (1). One

34 common avoidable source of UV radiation is an artificial tanning unit (hereafter referred to as

35 a sunbed).

36 Terrestrial UV radiation is classified into the UVA ( $315 \mathrm{~nm}$ to $400 \mathrm{~nm})$ and UVB (280 $\mathrm{nm}$ to

$37315 \mathrm{~nm}$ ), both of which cause damage to the skin (1).

38 The processes that lead from UV radiation exposure to skin cancer (photocarcinogenisis) are

39 complex and involve the interplay between various biochemical processes (3). UV radiation

40 reaching cells containing DNA (as illustrated in Figure 1) can damage DNA via several

41 mechanisms. When DNA absorbs UV radiation directly, chemical bonds can be altered,

42 producing DNA photoproducts. These include cyclobutane pyrimidine dimers (CPDs) and 43 pyrimidine (6-4) photoproducts (6-4 PPs). UV radiation can also form reactive oxygen species

44 (ROS) within the cell, which can cause chemical reactions that lead to DNA damage (3). CPDs

45 have been shown to form after exposure to UV has ceased in both melanin rich melanocytes

46 (4) and in keratinocytes containing no melanin (5). These are known as 'dark CPDs' and can

47 contribute up to half of the total CPD yield observed in melanocytes (4). The formation of UV-

48 induced DNA photoproducts is very common and on average each skin cell forms 50-100

49 photoproducts per second of sunlight exposure, though most occurrences are corrected by cell

50 repair processes $(6,7)$. If damage caused by a photoproduct is not corrected, the DNA sequence

51 can be miscopied, leading to a mutation. If this mutation occurs in a gene involved in cancer

52 formation, for example the tumour suppressor gene $\mathrm{p} 53$, this may have serious consequences 
53 (2,3). Previously only UVB has been considered to carry sufficient energy to cause damage to

54 cells, however UVA radiation has recently been shown to also be cytotoxic $(8,9)$.

55 The basal layer of the skin can accumulate enough DNA damage to lead to cancer (10). In

56 healthy skin, the basal layer (Layer 4 in Figure 1) produces skin cells, some of which undergo

57 a process of terminal differentiation, moving upwards, reaching the stratum corneum (Layer 1

58 in Figure 1) in about 2 weeks. The epidermal layer of skin (Layer 2 in Figure 1) can also

59 acquire DNA damage, however as the cells are committed to terminal differentiation, this is

60 unlikely to have serious consequences $(10,11)$.

61 The Monte Carlo Radiative Transfer (MCRT) method we apply uses localized probabilities

62 describing photon behavior to model the paths of many photon packets (hereafter referred to as

63 'power packets') through a scattering and absorbing medium. MCRT is well optimized to

64 model a complex structure such as the skin, as multiple physical quantities can be recorded

65 simultaneously, with desired spatial resolution limited only by the computational power

66 available. Multi-layered 3D MCRT grid codes have been extensively used for various

67 purposes in modelling light-tissue interactions (12-14). By applying MCRT to simulate photon

68 transport through skin tissue, the number of CPDs induced by UV irradiation can be estimated.

69 The aim of the work presented here is to quantify direct DNA damage in skin types I and II

70 caused by irradiation from a typical UK sunbed in comparison to the damage caused by a high

71 solar UV exposure, chosen as the solar spectrum from a cloudless day in July at midday in

72 Thessaloniki, Greece $(15,16)$. The spectra used are shown in Figure 2. The MCRT codes used

73 are an extension of codes developed by our group for modelling light transport in skin $(14,17)$.

74 MATERIALS AND METHODS 
75 A 3D grid was built for the MCRT simulation containing optical properties that could be varied on

76 a voxel by voxel basis allowing multiple layer skin structures to be simulated. By tracking the

77 progress of a power packet through the grid, and recording the energy absorbed by the DNA

78 present in the basal layer, the number of CPDs formed could be calculated.

79 The original FORTRAN 3D grid code used was developed for astronomy applications from a

80 publicly available code $(18,19)$ which was adapted and validated for tissue optics in previous

81 works $(14,17)$.

82 Geometry: The 3D grid for simulations is a cube of dimensions $1 \mathrm{~mm} \times 1 \mathrm{~mm} \times 1 \mathrm{~mm}$ and

83 contains $10^{6}$ cubic voxels of side $0.01 \mathrm{~mm}$. Within a single voxel the optical properties are

84 homogeneous. Each voxel is allocated specific optical properties depending upon its spatial

85 location, allowing the structure of skin to be simulated via the five-layer model shown in Figure 1.

87 In the model, the top layer of the skin, the stratum corneum (layer 1), has a flat surface and base.

88 Below this is the epidermal layer (layer 2) which has an undulating shape to represent the dermal

89 papillae. Layer 3 is a layer of melanised epidermis which, in non-UV adapted skin types I-II takes

90 the form of melanosomes residing above the basal layer (layer 4) $(20,21)$. This model simulates

91 skin types I-II by concentrating the melanin fraction above the basal layer (layer 4). Skin types III-

92 VI are not represented by this model; as in these skin types melanin is distributed throughout the

93 epidermis in varying concentrations. The final layer in this model (layer 5) is the dermis.

94 The maximum and minimum depth of each layer from the surface are shown in Figure 1, and the

95 average depth from the surface is shown in Table 1. The base of the stratum corneum (layer 1) is

96 considered to be flat, along with the base of the modelled dermis (layer 5) which marks the bottom

97 of the 3D grid. The base of the epidermis (layer 2), melanin layer (layer 3), and basal layer (layer 
98 4) are modeled using a 3D sinusoid to approximate the shape of dermal papillae (Equation 1)

99 where $z(x, y)$ is the surface of the layer in $\mathrm{mm}, \mathrm{x}$ and $\mathrm{y}$ are the horizontal coordinates, and the

100 depth is the average depth of each layer from the surface as listed in Table 1.

101

$$
z(x, y)=0.03 m m \times \sin (x / 0.015 \mathrm{~mm}) \times \sin y /(0.015 \mathrm{~mm})+\operatorname{depth}
$$

104 To simulate a layer of skin, repeating boundaries were implemented on the vertically oriented

105 faces of the grid. A power packet leaving the grid on a vertical face rejoins the grid on the

106 opposing vertical face; with all properties pertaining to the power packet other than position

107 retained. This simulates an infinitely repeating medium in the horizontal directions, and so

108 approximates a $1 \mathrm{~mm}^{2}$ section of a large area of skin of depth $1 \mathrm{~mm}$.

110 Irradiation: MCRT works by simulating the progress of one power packet at a time through the

111 medium. A power packet is initialized with a spatial location in the grid, an initial direction of

112 travel, and a single wavelength. To simulate irradiation from a broadband source, a wavelength is

113 obtained using a random sampling of the spectral irradiance of the source spectrum.

114 To quantify the DNA damage caused by a typical UK sunbed, a suitable UV source was chosen.

115 The sunbed spectrum was typical of those measured in a recent study that also found that over

$11690 \%$ of such devices exceeded the maximum effective irradiance recommended by the European

117 Commission $(23,24)$. A spectrum from Thessaloniki in Greece in midsummer at midday gives an

118 example of a natural environment with high UV exposure $(15,16)$. The spectra used in our

119 simulations are shown in Figure 2. 
121 The sunbed was considered a purely diffuse source, and the solar spectrum a mix of direct and

122 diffuse components determined by the latitude and longitude of the location on earth where the

123 spectrum was recorded (25). In this model, direct components are modelled as power packets with

124 a direction of entry normal to the surface of the grid, and diffuse components are modelled by

125 assigning a random direction of entry to the grid. For both sources, the initial position of the power

126 packet is randomly sampled to simulate uniform irradiation of the surface.

127 Due to the refractive index change between the air and the surface of the skin, Fresnel reflections

128 are taken into account at this boundary. Power packets leaving the simulated skin structure at the

129 lower face are terminated. Fresnel reflections between the layers within the skin are not accounted 130 for.

132 Absorption and Scattering: The path taken by a photon through the simulated tissue structure

133 is determined by the optical properties assigned to each voxel, comprising the absorption and

134 scattering coefficients $\mu_{a}$ and $\mu_{s}$, the refractive index $n$, and the scattering anisotropy factor $g$

135 assigned to individual voxels. In general, there is a high level of variability of optical properties

136 between individuals and from published literature $(26,27)$.

137 The upper epidermal layers (layers 1,2 and 3 in Figure 1) residing above the basal layer are

138 responsible for the majority of the attenuation of UV radiation reaching the basal layer. UV

139 radiation reaching the dermis is also scattered back to the basal layer; as there is no protective

140 melanin at the basal-dermal junction this represents an important component of the model.

141 The Henyey Greenstein phase function, $H G(\theta)$ used throughout the model is described by

142 Equation 2 and is used in conjunction with the anisotropy factor $g$ (defined as the average of 
$143 \cos \theta$ where $\theta$ is the angle between the direction of travel and the direction of scattering, described

144 by Equation 3) to model the angular scattering phase function, as skin is highly forward scattering

$145(28,29)$. The anisotropy factor $\mathrm{g}$ is wavelength dependent and is described by Equation 4

146 throughout the model, where the wavelength $\lambda$ is given in $\mathrm{nm}$. In Equation 2, $\theta$ is the scattering

147 angle and $g$ is the anisotropy factor $-1 \leq g \leq 1$.

148

$$
\begin{aligned}
& H G(\theta)=\frac{1}{4 \pi\left(1+g^{2}(\lambda)-2 g(\lambda) \cos \theta\right)^{\frac{3}{2}}} \\
& g(\lambda)=\langle\cos \theta\rangle=\int_{0}^{\pi} H G(\theta) \cos \theta 2 \pi \sin \theta d \theta \\
& g(\lambda)=0.62+0.29 \lambda \times 10^{-3}
\end{aligned}
$$

150 Within our work, the $g$ values vary linearly between 0.7 and 0.74 as described by Equation 4 and

151 the refractive index of tissue $n=1.38$.

152 The optical properties used to characterize the stratum corneum and the epidermal layers are from

153 data published by Van Gemert et al. (29) who derived absorption and scattering spectra by

154 transforming experimentally determined transmittance and reflectance spectra.

155 The stratum corneum provides significant protection against UV radiation, due to the strong

156 preference for absorption and scattering in the UVB; although absorption and scattering is high

157 throughout the full UV spectrum. It is assumed the stratum corneum contains no melanin in skin

158 types I and II, and as such the skin type has no influence on the absorption or scattering properties.

159 The wavelength dependent absorption for the stratum corneum is shown in Figure 3; and the

160 wavelength dependent scattering spectrum is shown in Figure 4. 
164 The epidermis is the layer of the skin above the basal layer, and as such, the scattering and 165 absorption of UV radiation within the epidermis has a significant effect on the amount of UV 166 radiation absorbed by the basal layer. The epidermal layer also contains living cells that are 167 susceptible to UV induced DNA damage. The properties used to model this layer are from the 168 properties published by Van Gemert et al. (29). The original experimental data were measured 169 using 'medium complexioned Caucasian skin' and the epidermal absorption coefficients reported 170 describe all the sum total of absorption coefficients due to all chromophores present in skin tissue.

171 Using melanin concentrations described by Karsten et al. (20), it was assumed the experimental 172 data described skin with a melanin volume fraction $V_{m e l}$ of $4 \%(33,34)$. This contribution is 173 removed from the epidermal layer (layer 2) to simulate epidermis without melanin; as shown in

174 Figure 3. The skin type dependent contribution of melanin to the absorption of the epidermis is 175 calculated and concentrated in the melanin layer (layer 3).

176 Melanin is the primary chromophore responsible for shielding the DNA-containing basal layer 177 from DNA damage (35). The estimated volume fraction of melanin present in the skin $V_{m e l}$ is skin

178 type dependent, and ranges from 0-3\% for skin type I, and from 3-5\% for skin type II $(20,21,36)$.

179 Values of $2 \%$ for skin type I and 4\% for skin type II were chosen. In the skin, melanin is created

180 by melanocytes in the form of melanosomes, and is present in two types (eumelanin is a brown or

181 black pigment, and pheomelanin is a red pigment). Melanosomes are taken up by keratinocytes, 182 where they cluster around the nucleus, shielding DNA. To simulate this distribution of melanin, a 183 single layer of melanin is modelled above the basal layer, shown in Figure 1. To model the optical 184 properties for skin types I-II, the absorption resulting from the melanin volume fraction 
185 corresponding to the skin type was added to the epidermal optical properties; as shown in Figure

186 3. The optical properties resulting from a combination Eumelanin and pheomelanin are modelled

187 using Equation 5 (32), where the wavelength $\lambda$ is given in $\mathrm{nm}, V_{m e l}$ is the volume fraction of

188 melanin in the tissue, and the absorption coefficient $\mu_{a, m e l}$ is given in $\mathrm{cm}^{-1}$.

189

$$
\mu_{a, m e l}(\lambda)=6.6 \times 10^{11} \lambda^{-3.33} V_{m e l}
$$

191 In the model, the contribution of melanin to the epidermal layer absorption coefficient is removed

192 from layer 2 and concentrated in a single layer of voxels (layer 3) sited directly above the DNA

193 containing basal layer (layer 4). These absorption coefficients are shown in Figure 3. The

194 scattering coefficient $\mu_{s}$ (given in $\mathrm{cm}^{-1}$ ) for the epidermal and melanin layers are considered to

195 exhibit the same wavelength dependence, as shown in Figure 4.

196 The epidermal layer and the basal layer both contain DNA. DNA is a strong absorber of UVB

197 radiation, as indicated in Figure 3. The extinction coefficient spectrum of oligomeric duplex

198 dA20:dT20 has previously been used to determine photo-damage in DNA (31) and is used here as

199 an approximation for the absorption coefficient of the DNA contained within cells. The

200 concentration of DNA within the epidermal and basal layers is estimated using a volumetric

201 method adapted from Mohlenhoff et al (30). Using the number of bases per human diploid cell

202 (12.8 billion bases) along with average cell sizes for cells in the basal layer and epidermis (on

203 average $13 \mu \mathrm{m}$ and $20 \mu \mathrm{m}$ respectively (37)), cells in the epidermal layers (layers $2 \& 3$ ) are

204 estimated to have a DNA concentration of approximately 0.005 moles per liter, and those in the

205 basal layer to have a DNA concentration of 0.018 moles per liter.

206 Equation 6 is used to combine these concentrations with the extinction coefficient per base taken 
207 from Mouret et al. (31) to retrieve the absorption coefficient $\mu_{a, D N A}$ (where the wavelength $\lambda$ is

208 given in $\mathrm{nm}$ and $\mu_{a, D N A}$ is given in $\mathrm{cm}^{-1}$ ). The DNA absorption is shown in Figure 3 for layers 2,

2093 and 4. The scattering in the basal layer (layer 4) is considered to follow the wavelength

210 dependent form described by Equation 7 (32), where $\lambda$ is given in $\mathrm{nm}$ and $\mu_{s}$ is given in $\mathrm{cm}^{-1}$.

211

$$
\begin{gathered}
\mu_{a, D N A}(\lambda)=\log _{e}(10) \epsilon_{D N A}(\lambda) C_{D N A} \\
\mu_{s}(\lambda)=1.752 \times 10^{8} \lambda^{-2.33}+134.67 \lambda^{-0.494}
\end{gathered}
$$

212

213 The Dermis is the deepest layer of skin included in our model. Equation 8 (32)

214 describes $\mu_{a, \text { dermis }}$ (where $\mu_{a, \text { dermis }}$ is given in $\mathrm{cm}^{-1}$ and the wavelength $\lambda$ is given in $\mathrm{nm}$ ). 215

$$
\begin{gathered}
\mu_{a, \text { dermis }}(\lambda)=\left(1-f_{\text {blood }}\right) \mu_{\text {base }}(\lambda)+f_{\text {blood }} \mu_{a, \text { blood }}(\lambda) \\
\mu_{a, \text { blood }}(\lambda)=\frac{1}{\omega_{\text {hgb }}} \log _{e}(10)\left(\epsilon_{a, \text { ohb }}(\lambda) S O_{2}+\epsilon_{a, d h b}(\lambda)\left(1-S O_{2}\right)\right) C_{h b} \\
\mu_{\text {base }}(\lambda)=7.84 \times 10^{7} \times \lambda(n m)^{-3.255}
\end{gathered}
$$

217 To determine the absorption coefficient for blood $\mu_{a, b l o o d}(\lambda)$ as used in Equation 8, Equation 9 is 218 used. The hemoglobin concentration $C_{h b}$ of $150 \mathrm{gL}^{-1}$ and an oxygen saturation $\mathrm{SO}_{2}$ of $75 \%$ are 219 used in Equation 9 (where $\lambda$ is given in $\mathrm{nm}$ ); and the extinction coefficients for hemoglobin are 220 taken from Jacques et al. (32). The molecular weight of hemoglobin $\omega_{h g b}$ is $64458 \mathrm{~g} / \mathrm{mol}$. The

221 background absorption, $\mu_{\text {base }}(\lambda)$ is taken from Jacques et al. (32).

222 The absorption coefficient $\mu_{a, b l o o d}(\lambda)$, is shown in Figure 3 . The scattering coefficient is 223 equivalent to that described for the basal layer by Equation 7 and shown in Figure 4. 
224 The MCRT model allows recovery of the number of absorbed photons and the wavelength

225 dependent fluence rate in all the voxels in the model. Using this, the number of photons absorbed

226 by the basal layer can be determined. For a photobiological process, such as CPD formation, the

227 efficiency may be described by the quantum yield, $\phi$, expressed as

228

$$
\phi=\frac{N_{\text {process }}}{N_{\text {absorbed }}}
$$

230 Where $N_{\text {process }}$ is the number of photons causing the biological effect and $N_{\text {absorbed }}$ is the total

231 number of absorbed photons. Banyasz et al. demonstrated quantum yields for CPD formation to be

2320.05 for UVB and 0.0005 for UVA (38). The number of absorbed photons for sunlight and sunbed

233 sources were evaluated and compared. To attempt to quantify the additional risk to human health

234 of sunbed use, CPDs and photons absorbed due to sunbed irradiation are expressed as a proportion

235 of those absorbed due to solar irradiation, as risks to health from solar irradiation are well

236 characterized (15).

\section{RESULTS}

238 Figure 5 shows maps detailing the total number of absorbed photons per second through a

239 central slice of the 3D grid for both the Mediterranean sun and the sunbed for skin type I and

240 skin type II. The maps clearly show the high absorption by melanin residing above the basal

241 layer; and that more photons are absorbed by the grid simulating sunbed irradiation. Spotting

242 at the base of the grids is characteristic Monte Carlo noise. A running total for energy absorbed

243 by each voxel in the grid as a function of wavelength is recorded throughout the simulation. In 
244 the map presented in Figure 5, the total number of photons is presented (obtained using 245 Equation 12, and summing over all wavelengths), and no wavelength dependent information 246 remains.

$$
N_{\lambda}=\frac{E_{\lambda, \text { voxel }}}{E_{\lambda, \text { photon }}}=\frac{\lambda E_{\lambda, \text { voxel }}}{h c}
$$

Table 2 presents results obtained when the depth of the epidermal layer is varied,

251 showing as the depth of the epidermis is increased, the ratio of CPDs formed within the basal

252 layer increases, while the total ratio of photons absorbed decreases. The wavelength dependent

253 energy absorbed within the basal layer was isolated, and using the quantum yields for CPD

254 formation of 0.05 for UVB and 0.0005 for UVA (38) with Equation 11, the number of CPDs

255 formed is retrieved. The number of CPDs formed by the sunbed is expressed as a fraction of 256 those formed by the Medditeranean sun.

258 An example of the proportions of CPDs formed within the basal layer with respect to skin type

259 and UV band is shown in Table 3. The number of CPDs formed by the sunbed is expressed as 260 a fraction of those formed by the Medditeranean sun.

262 Figure 6 shows the fluence incident on the basal layer for both skin types and both radiation 263 sources. Throughout the simulation, the fluence incident on each voxel is recorded for the 
264 whole grid. The fluence incident on the voxels corresponding to the basal layer is extracted

265 and shown in Figure 6.

267 CPDs are not just formed in the basal layer of the skin, as cells in the epidermis (layers $2 \& 3$

268 in Figure 1) also contain DNA. The numbers of CPDs formed per $1 \mathrm{~mm}^{2}$ skin comprising 269 epidermis and basal layer is presented in Table 4.

\section{DISCUSSION}

272 Previous work suggests that $90 \%$ of sunbeds in the UK emit UV levels exceeding current EU

273 recommended limits (23). On-site sunbed measurements were used to evaluate exposure

274 scenarios. Further study quantified the increased risk in developing squamous cell carcinoma

275 by age 55 years in terms of additional doses of UV radiation due to sunbed use between the 276 ages of 20-35 years. The lowest $5^{\text {th }}$ percentile of additional UV exposure (corresponding to 83

277 standard erythemal doses) increased the risk of developing squamous cell carcinoma by $40 \%$.

278 At the $95^{\text {th }}$ percentile of additional exposure (corresponding to 302 standard erythemal doses)

279 the risk had increased by $300 \%$ (15).

280 In the present study, MCRT modelling was used to simulate the transmission of UV radiation

281 through the upper layers of skin tissue. We used published optical properties in a five-layer

282 model comprising the stratum corneum, non melanised epidermis, a melanin layer, the basal

283 layer (in which skin stem cells reside) and the dermis. The basal layer and the protective 
284 melanin were modelled with a 3D sinusoidal surface as an approximation to the undulating 285 shape of the dermal papillae folds.

286 MCRT modeling to simulate UV transport through skin tissue allows complete control over 287 parameters such as the optical properties; in contrast to laboratory experiments where optical 288 properties would likely vary within and across individuals. MCRT also allows wavelength 289 dependent results to be extracted from selected tissue depths irradiated with real-world 290 sources, such as the sun, without attempting to simulate such wavelength dependence with a 291 monochromator. A model of this type is currently unable to fully simulate the many pathways 292 by which UV radiation causes damage to skin. CPDs are often found within signature 293 mutations in UV induced non melanoma skin cancers (SCCs and BCCs) that are linked to 294 lifetime cumulative UV exposure (15), and as such the CPD yield was chosen as an indicator 295 for DNA damage. However the model does not include UV induced enzymatic DNA repair 296 mechanisms (which would reduce the total number of CPDs formed), nor other sources of 297 CPD formation, such as those formed by ROS initiated by UV absorption (3), or dark CPDs 298 formed both with and in the absence of melanin (4,5). As a result, the model is likely to 299 underrepresent the total number of CPDs formed and by way of compensation, ratios of 300 sunbed induced CPDs to solar induced CPDs are presented. It may be possible to extend the 301 model to take these processes into account by adding a time dependent aspect to the model 302 following initial irradiation, allowing repair processes to be modelled along with dark CPDs.

303 This model is only applicable to Fitzpatrick skin types I-II that are not UV adapted, where the 304 melanin resides immediately above the basal layer; however the model could be adapted to 305 simulate other skin types. As part of the response to UV radiation, melanin moves up into the 306 epidermal layer and more melanin is created by melanosomes in the basal layer (35); although 
307 research indicates that the role of melanin can no longer be considered as purely protective,

308 and it may in fact be carcinogenic (4). When skin is not UV adapted, the UV radiation causes

309 greater risk to skin health due to the lower concentration of melanin in the epidermis. However

310 what we have done is to quantify, for the first time, the relative risk of direct CPD formation

311 from sunbed use compared to solar exposure. By implication this quantifies the risk of direct

312 DNA damage due to exposure to UV radiation from a sunbed in comparison to that from

313 Mediterranean sunlight.

314 By utilizing the optical properties for DNA and the quantum yield for CPDs the number of

315 CPDs formed was calculated. The simulation was run with a typical sunbed spectral irradiance

316 and, for comparison, solar irradiance. Both sunbeds and sunlight are primarily UVA sources;

317 and direct CPDs are primarily formed by UVB; indicating that true CPD yields, including

318 those due to ROS and dark CPDs $(4,5)$ are likely to be higher than those stated here. Table 4

319 details for skin type I that for solar irradiation, UVB radiation results in a higher proportion of

320 CPDs formed in the basal layer than for UVA radiation, even though only $0.5 \%$ of UVR

321 radiation incident on the basal layer is UVB (Figure 6). However, for sunbed irradiation, UVA

322 radiation is responsible for the majority of CPDs formed in the basal layer. This is an

323 important finding, as it shows that even though the yield for UVA CPDs is 100 times less than

324 that for UVB (38), the high UVA output of the sunbed results in the majority of CPDs being

325 formed due to UVA radiation.

326 Table 4 also details the total number of CPDs formed in the epidermis for the skin type I

327 sample. For solar and sunbed UVA irradiation, there are about 4.6 times more CPDs formed in

328 the epidermis than in the basal layer respectively. For solar and sunbed UVB radiation, there

329 are about 7 times more CPDs formed in the epidermis than in the basal layer. Although the 
330 DNA concentration in the basal layer is approximately 4 times higher than that in the

331 epidermis; there are 8 times as many voxels comprising the epidermis than comprising the

332 basal layer. This, coupled with the fact that the epidermis is closer to the surface, and the small

333 penetration depth of UVB coupled with the high UVB CPD yield explains why so many more

334 CPDs are formed in the epidermis than in the basal layer. As keratinocytes in the epidermis are

335 likely to be committed to terminal differentiation, any damage accumulated here is unlikely to

336 have long term consequences $(10,11)$. As a result, it may be that DNA within the epidermis

337 actually plays a protective role in shielding the basal layer from UV radiation.

338 Figure 6 shows the fluence incident on the basal layer for both skin types and both irradiation

339 sources. In both cases, radiation below $315 \mathrm{~nm}$ (UVB), reaches the basal layer although in

340 small amounts. UVB is strongly absorbed by DNA (30) and UVB CPDs have a high quantum

341 yield (31). The basal layer has a high concentration of DNA (37) and is considered the layer of

342 the skin that can accumulate enough DNA damage to cause risk to human health (10). The

343 argument that UVB radiation can not penetrate far enough into the epidermis to cause

344 significant damage to the basal layer is not supported by this model. However, it is important

345 to note that for solar radiation, the incident radiation contains $3 \% \mathrm{UVB}$, and for the sunbed,

346 the incident radiation contains $1.4 \%$ UVB (see Figure 2). For both sunbed and solar

347 irradiation, of the total fluence incident on the basal layer, the percentage of UVB radiation is

348 greatly reduced from that incident on the skin surface (see Figure 6) This is due to the

349 shielding effects of the upper layers of the epidermis, and the wavelength dependent

350 absorption by the upper layers of skin.

351 Absolute numbers of CPDs formed in the basal layer differ between the skin types, as shown

352 in Figure 5, Table 4, and indicated by the difference in fluence rate in the basal layer (see 
353 Figure 6). However, if the risk of DNA damage from sunbed use for one skin type can be

354 deduced from the ratio between the number of CPDs formed due to sunbed exposure to the

355 number formed due to solar exposure, then Table 3 indicates that for both skin types, the

356 additional risk is almost equal; despite skin type II containing twice as much melanin $(20,21)$.

357 Increasing the depth of the epidermal layer appears to gradually increase the ratio between

358 sunbed and solar CPD formation, despite the ratio of photons absorbed decreasing. This is due

359 to UVA penetrating deeper than UVB; and continuing to produce CPDs. This indicates that

360 UVA radiation may also affect live cells residing in layers of the skin deeper than the basal

361 layer.

\section{Conclusion}

363 When skin is irradiated with a sunbed, UVA photons make a large contribution to CPD

364 formation. For both sources, UVA radiation contributes significantly to the total amount of

365 CPDs formed. This is despite UVB being preferentially absorbed by DNA compared to UVA,

366 and also forming more CPDs per absorbed UVB photon than per absorbed UVA photon, and

367 despite UVB being generally considered to be the part of the UV spectrum to present the 368 greater risk to human health. We estimate that for skin type I, 12 min on a typical sunbed

369 produces approximately the same amount of DNA damage as 30 min sunbathing in the midday

370 Mediterranean summer sun. We present similar results for CPD formation ratios for both skin

371 types, indicating that the relative increase in DNA damage from sunbed use may be the same

372 for both skin types I and II.

373 ACKNOWLEDGMENTS: Isla Rose Mary Barnard acknowledges financial support

374 from an UK EPRSC PhD studentship EP/N509759/1. 


\section{REFERENCES}

1. Narayanan, D. L., R. Saladi and J. Fox (2010) Ultraviolet radiation and skin cancer. Int J Dermatol. 49, 978-86.

2. Leiter, U. and C. Garbe (2008) Epidemiology of melanoma and non melanoma skin cancer- the role of sunlight. Adv Exp Med Biol. 624, 89-103.

3. Black, H. S. and F. deGruijl (1997) Photocarcinogenesis: an overview. J Photochem Photobio B. 40, 29-47.

4. Premi, S., S. Wallisch, C. M. Mano, A. B. Weiner, A. Bacchiocchi, K. Wakamatsu, and D. E. Brash (2015) Chemiexcitation of melanin derivatives induces DNA photoproducts long after UV exposure. Science. 347(6224), 842-847.

5. Delinasios, G. J., M. Karbaschi, M. S. Cooke and A. R. Young (2018) Vitamin E inhibits the UVAI induction of "light" and "dark" cyclobutane pyrimidine dimers, and oxidatively generated DNA damage, in keratinocytes. Sci Rep. 8(1), 423.

6. Goodsell, D. S. (2001) The molecular perspective: ultraviolet light and pyrimidine dimers. The Oncologist. 6(3), 298-299. repair. Science. 286(5446), 1897-1905. 
393 8. Huang, X. X., F. Bernerd and G. M. Halliday (2009) Ultraviolet A within sunlight 394 induces mutations in the epidermal basal layer of engineered human skin. Am J Pathol. 174(4), 1534-1543.

9. Boniol, M., P. Autier, P. Boyle and S. Gandini (2012) Cutaneous melanoma attributable to sunbed use: systematic review and meta-analysis. BMJ. 345, e4757.

10. Owens D. M., and F. M. Watt (2003) Contribution of stem cells and differentiated cells to epidermal tumors. Nat Rev Cancer. 3, 444-451.

11. Young, A. R., G. I. Harrison, C. A. Chadwick, O. Nikaido, J. Ramsden, and C. S. Potten (1998) The similarity of action spectra for thymine dimers in human epidermis and erythema suggests that DNA is the chromophore for erythema. J Invest Dermatol. 111(6), 982-988.

404 12. Wang, L., S. L. Jacques and L. Zheng (1995) MCML-Monte Carlo modeling of 405 light transport in multi-layered tissues. Comput Methods Programs Biomed. 47(2),

13. Boas, D. A., J. P. Culver, J. J. Stott and A. K. Dunn (2002) Three dimensional Monte Carlo code for photon migration through complex heterogeneous media including the adult human head. Opt express. 10(3), 159-170. Moseley (2015) 3D Monte Carlo radiation transfer modelling of photodynamic therapy. Proc SPIE. 9531(Biophotonics South America). 
413 15. Tierney, P., F. R. Gruijl, S. Ibbotson and H. Moseley (2015) Predicted increased 414 risk of squamous cell carcinoma induction associated with sunbed exposure habits. Br J Dermatol. 173(1), 201-208.

16. Zempila M., J. H. G. M. van Geffen, M. Taylor, I. Fountoulakis, M. Koukouli, M. van Weele, R. J. van der A, A. Bais, C. Meleti and D. Balis (2017) TEMIS UV product validation using NILU-UV ground-based measurements in Thessaloniki, Greece. Atmos Chem Phys, 17, 7157-7174.

17. Campbell, C. L., K. Wood, R. M. Valentine, C. T. A. Brown and H. Moseley (2015) Monte Carlo modelling of daylight activated photodynamic therapy. Phys Med Biol. 60(10), 4059.

18. Wood, K. and R. J. Reynolds (1999) A model for the scattered light contribution and polarization of the diffuse Ha galactic background. ApJ. 525(2), 799.

19. Wood, K., L. M. Haffner, R. J. Reynolds, J. S. Mathis and G. Madsen (2005) Estimating the porosity of the interstellar medium from three-dimensional photoionization modeling of Hii regions. ApJ. 633(1), 295

20. Karsten, A. E., and J. E. Smit (2012). Modeling and verification of melanin concentration on human skin type. Photochem Photobiol. 88(2), 469-474. through VI. Arch Dermatol. 124(6), 869-871. 
constitutive photosensitivity. Photodermatol Photoimmunol Photomed. 13(4), 153158

436

23. Tierney, P., J. Ferguson, S. Ibbotson, R. Dawe, E. Eadie and H. Moseley (2013) Nine out of 10 sunbeds in England emit ultraviolet radiation levels that exceed current safety limits. Br J Dermatol. 168(3), 602-608.

24. European commission scientific committee on consumer products (2006) Preliminary opinion on biological effects of ultraviolet radiation relevant to health with particular reference to sun beds for cosmetic purposes. European Commission.

25. Bird, R. E. (1984) A simple, solar spectral model for direct-normal and diffuse horizontal irradiance. Solar energy. 32(4), 461-471.

26. Lister, T., P. A. Wright and P. H. Chappell (2012) Optical properties of human skin. J Biomed Opt. 17(9), 0909011-09090115.

27. Jacques, S. L. (2013). Optical properties of biological tissues: a review. Phys Med Biol. 58(11), R37.

28. Bruls, W. A. and J. C. Van Der Leun (1984) Forward scattering properties of human epidermal layers. Photochem Photobiol. 40(2), 231-242.

29. Van Gemert, M. J., S. L. Jacques, H. J. Sterenborg and W. M. Star (1989) Skin optics. IEEE Trans Biomed Eng. 36(12), 1146-1154.

30. Mohlenhoff, B., M. Romeo, M. Diem and B. R. Wood (2005) Mie-type scattering and non-Beer-Lambert absorption behavior of human cells in infrared microspectroscopy. Biophys J, 88(5), 3635-3640. 
455 31. Mouret, S., C. Philippe, J. Gracia-Chantegrel, A. Banyasz, S. Karpati, D. Markovitsi and T. Douki (2010) UVA-induced cyclobutane pyrimidine dimers in DNA: a direct photochemical mechanism? Org Biomol Chem. 8(7), 1706-1711.

32. Jacques, S. L. (1998) Skin optics. Oregon Medical Laser Center News 1998. 1, 1-9.

33. Wan, S., R. Anderson and J. A. Parrish (1981) Analytical modeling for the optical properties of the skin with in vitro and in vivo applications. Photochem Photobiol. 34(4), 493-499.

34. Everett, M. A., E. Yeargers, R. M. Sayre and R. L. Olson (1966) Penetration of epidermis by ultraviolet rays. Photochem Photobiol. 5(7), 533-542.

35. Brenner, M., and V. J. Hearing (2008). The protective role of melanin against UV

36. Iglesias-Guitian, J. A., C. Aliaga, A. Jarabo and D. Gutierrez (2015) A biophysically-based model of the optical properties of skin aging. Computer Graphics Forum. 34(2), 45-55.

37. Watt, F. M., and H. Green (1981) Involucrin synthesis is correlated with cell size in Markovitsi (2012) Electronic excited states responsible for dimer formation upon UV absorption directly by thymine strands: joint experimental and theoretical study. J Am Chem Soc. 134(36), 14834-14845. 


\begin{tabular}{|c|c|c|}
\hline Layer Number & Skin Layer & $\begin{array}{c}\text { Average Depth from Surface } \\
(\mathrm{mm})\end{array}$ \\
\hline 1 & Stratum Corneum & 0.02 \\
\hline 2 & Epidermis & 0.08 \\
\hline 3 & Melanin Layer & 0.09 \\
\hline 4 & Basal Layer (DNA Layer) & 0.10 \\
\hline 5 & Dermis & (base of grid) \\
\hline
\end{tabular}

476 Table 1. Average depth of each layer from the surface of the 3D grid taken from Lock-

477 Anderson et al (22). Layer 1 (stratum corneum) and Layer 5 (dermis) both have flat bases. The 478 average depths listed for the epidermis (layer 2), the melanin layer (layer 3) and the basal layer 479 (layer 4) are added to Equation 1 to model the shape shown in Figure 1.

\begin{tabular}{|c|c|c|}
\hline $\begin{array}{c}\text { Epidermis/Basal/Dermis } \\
\text { Depths }(\mu \mathrm{m})\end{array}$ & $\begin{array}{c}\text { Photons Absorbed } \\
\text { Sunbed/solar }\end{array}$ & $\begin{array}{c}\text { CPDs } \\
\text { Sunbed/Solar }\end{array}$ \\
\hline $70 / 80 / 90$ & 4.17 & 2.48 \\
\hline $80 / 90 / 100$ & 4.16 & 2.50 \\
\hline $90 / 100 / 110$ & 4.15 & 2.65 \\
\hline $100 / 110 / 120$ & 4.16 & 2.74 \\
\hline $110 / 120 / 130$ & 4.15 & 2.75 \\
\hline
\end{tabular}

481 Table 2. Ratios of photons absorbed between sunbed and solar irradiation, and CPDs created, 482 for skin type I with varying epidermal depths, to approximate sites on the body of varying 483 epidermal depths. The $20 \mu \mathrm{m}$ depth of the stratum corneum is maintained, as is the $10 \mu \mathrm{m}$ 484 separation between the epidermal, melanin and basal layers (layers 2, 3 and 4), and the depth 485 of the epidermis is varied from $70 \mu \mathrm{m}$ to $110 \mu \mathrm{m}$. The ratio of CPDs formed is always smaller 
486 than the ratio of absorbed photons. This is due to the strong UVA irradiance from the sunbed, 487 which has a much smaller quantum yield for CPDs. 488

\begin{tabular}{|c|c|c|}
\hline UV Band & $\begin{array}{c}\text { Skin Type I CPDs } \\
\text { Sunbed/Solar }\end{array}$ & $\begin{array}{c}\text { Skin Type II CPDs } \\
\text { Sunbed/Solar }\end{array}$ \\
\hline UVA & 4.21 & 4.16 \\
\hline UVB & 1.68 & 1.67 \\
\hline
\end{tabular}

489 Table 3. Proportion of CPDs formed with respect to the band of the UV spectrum examined

490 for skin types I and II. The geometry used is as described in Figure 1, with average depths of

$49180 \mu \mathrm{m}$ for the epidermis, $90 \mu \mathrm{m}$ for the DNA layer and $100 \mu \mathrm{m}$ for the dermis. Little variation

492 is observed between skin types in the ratios of sunbed to solar UVB induced CPDs, however

493 there is a more marked difference in the ratios of sunbed to solar UVA induced CPDs.

\begin{tabular}{|c|c|c|c|c|}
\hline \multirow{2}{*}{ UV Band } & \multicolumn{2}{|c|}{ CPDs per second per mm ${ }^{2}$ (Solar) } & \multicolumn{2}{c|}{ CPDs per second per $\mathrm{mm}^{2}$ (Sunbed) } \\
\hline & Basal Layer & Epidermis & Basal Layer & Epidermis \\
\hline UVA & $2.41 \times 10^{7}$ & $1.10 \times 10^{8}$ & $1.01 \times 10^{8}$ & $4.76 \times 10^{8}$ \\
\hline UVB & $5.01 \times 10^{7}$ & $3.52 \times 10^{8}$ & $8.43 \times 10^{7}$ & $6.12 \times 10^{9}$ \\
\hline
\end{tabular}

495 Table 4. Absolute numbers of CPDs formed with respect to the band of the UV spectrum and

496 skin layer for skin type II. The geometry used is as described in Figure 1, with average depths 497 of $80 \mu \mathrm{m}$ for the epidermis, $90 \mu \mathrm{m}$ for the DNA layer and $100 \mu \mathrm{m}$ for the dermis. There are 49810304 voxels in the basal layer (layer 4), and 61976 voxels in the epidermal layers (layers 2 499 and 3). 


\section{FIGURE CAPTIONS}

501 Figure 1. The five-layer skin model used in the simulation; comprising the stratum corneum

502 (layer 1), the epidermis (layer 2), melanin-containing epidermis (layer 3), the basal layer (layer

503 4) and the dermis (layer 5). The figure shows the characteristic egg box sinusoidal pattern as

504 described by Equation 1, which is used to simulate dermal papillae. The inset summarizes the

505 maximum and minimum layer separations chosen to represent the geometry of the skin layers

506 modelled. For color, please see online version.

507 Figure 2. Spectra used to simulate irradiation of the skin. In (a) the broken line shows the

508 UV spectrum from a typical sunbed (where the spikes indicate characteristic mercury emission

509 lines) and the solid line shows a solar spectrum, shown alone in inset (b). This spectrum is

510 taken from a cloudless day in July at midday from Thessaloniki in Greece using ground based

511 instrumentation $(15,16)$. UVB radiation is defined as $280 \mathrm{~nm}$ to $315 \mathrm{~nm}$ and UVA radiation as

$512315 \mathrm{~nm}$ to $400 \mathrm{~nm}$. The solar spectrum has a total irradiance of $50.5 \mathrm{Wm}^{-2}$ with a UVB

513 irradiance of $1.5 \mathrm{Wm}^{-2}$ and a UVA irradiance of $49.0 \mathrm{Wm}^{-2}$. The sunbed spectrum has a total

514 irradiance of $283.0 \mathrm{Wm}^{-2}$ with a UVB irradiance of $4.0 \mathrm{Wm}^{-2}$ and a UVA irradiance of 279.0

$515 \mathrm{Wm}^{-2}$.

516 Figure 3. Modelled absorption coefficients as a function of wavelength (nm) as described in

517 the text for the five layer skin model, comprising a) the stratum corneum (layer 1), taken

518 directly from Van Gemert et al. (29) b) epidermis with melanin removed (layer 2), and 519 melanin layer (layer 3), derived using Equation 5 and data from Van Gemert et al. (29), c)

520 DNA absorption coefficients for layers 2,3 and 4 derived using the method described by 
521 Molenhoff et al. (30) and data from Mouret et al. (31) and d) the dermis, using Equations 8 and

5229 , as described by Jacques et al. (32).

523 Figure 4. Scattering coefficients as a function of wavelength $(\mathrm{nm})$ of a) the stratum

524 corneum (layer 1), taken directly from Van Gemert et al. (29) and b) the epidermal and 525 melanin layer (layers 2 and 3), taken directly from (29) and the basal and dermal layers (layers

5263 and 4) as described by Equation 7 derived by Jacques et al. (32).

527 Figure 5. Maps of photons deposited in the central slice of the model in terms of number of 528 absorbed photons per $\mathrm{cm}^{3}$ per second for both skin types. The top of each map shows a bright

529 flat layer, indicating strong absorption in the stratum corneum (layer 1). The bright sinusoidal 530 layer indicates high absorption of UVR in the melanin layer (layer 3), below which darker 531 spots indicate a lower overall level of absorption in the basal layer (layer 4). Dark spotting at 532 the base of layer 5 (the dermis) is characteristic Monte Carlo noise. For color, please see online 533 version.

534 Figure 6. Radiation incident on the basal layer for a) the solar simulation and b) the sunbed 535 simulation. In both skin types, and for both sources, a small amount of UVB radiation 536 penetrates as far as the basal layer. For a), in skin type I, $99.4 \%$ of fluence reaching the basal 537 layer is UVA, and $0.6 \%$ is UVB. For skin type II, $99.5 \%$ of solar fluence reaching the basal 538 layer is UVA, and $0.5 \%$ is UVB. For b) in both skin types I and II, $99.7 \%$ of fluence reaching 539 the basal layer is UVA, and $0.3 \%$ is UVB. Figure 2 shows the incident solar radiation contains $54097 \%$ UVA and $3 \%$ UVB, and the incident sunbed radiation contains $98.6 \%$ UVA and $1.4 \%$ 541 UVB. 


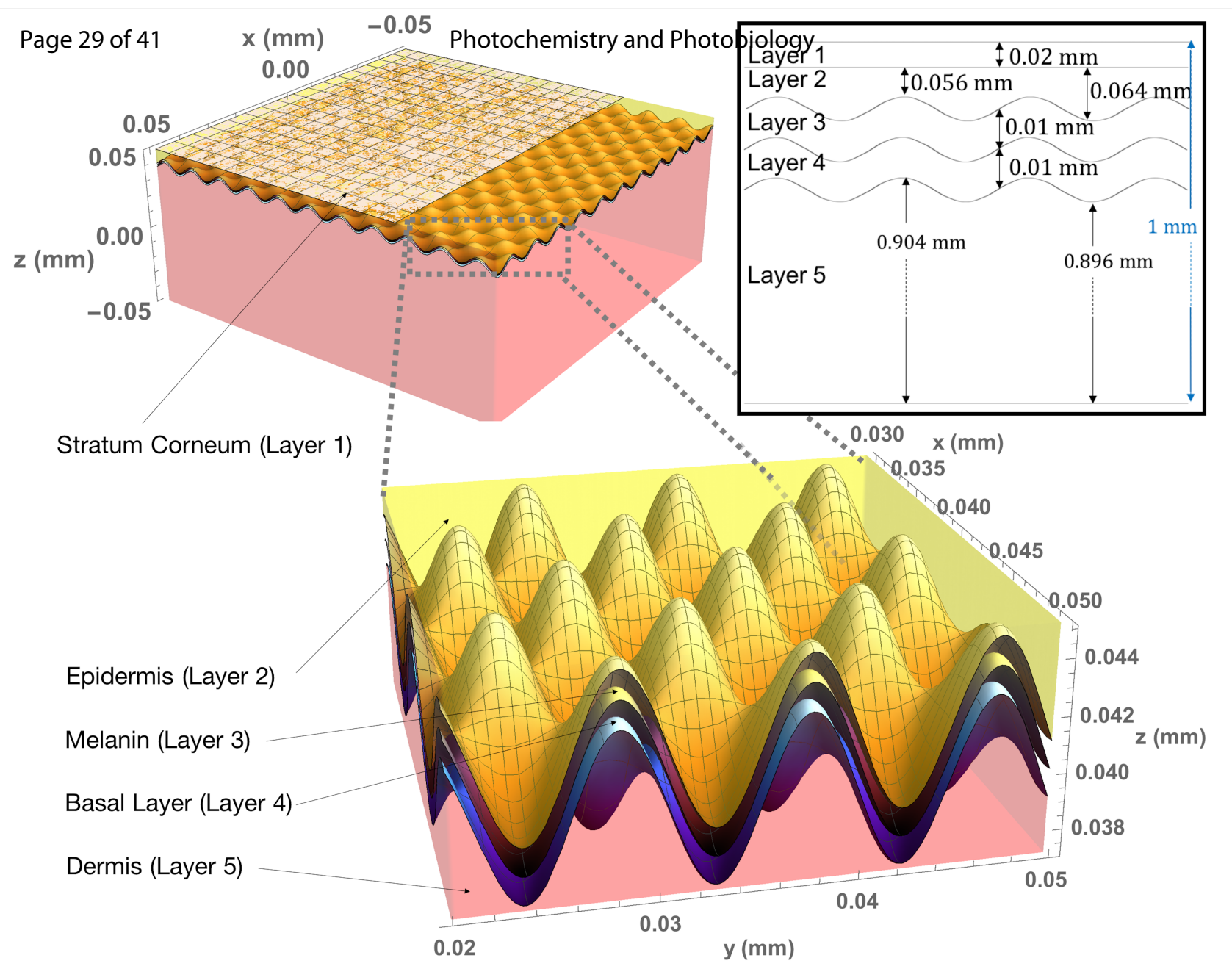




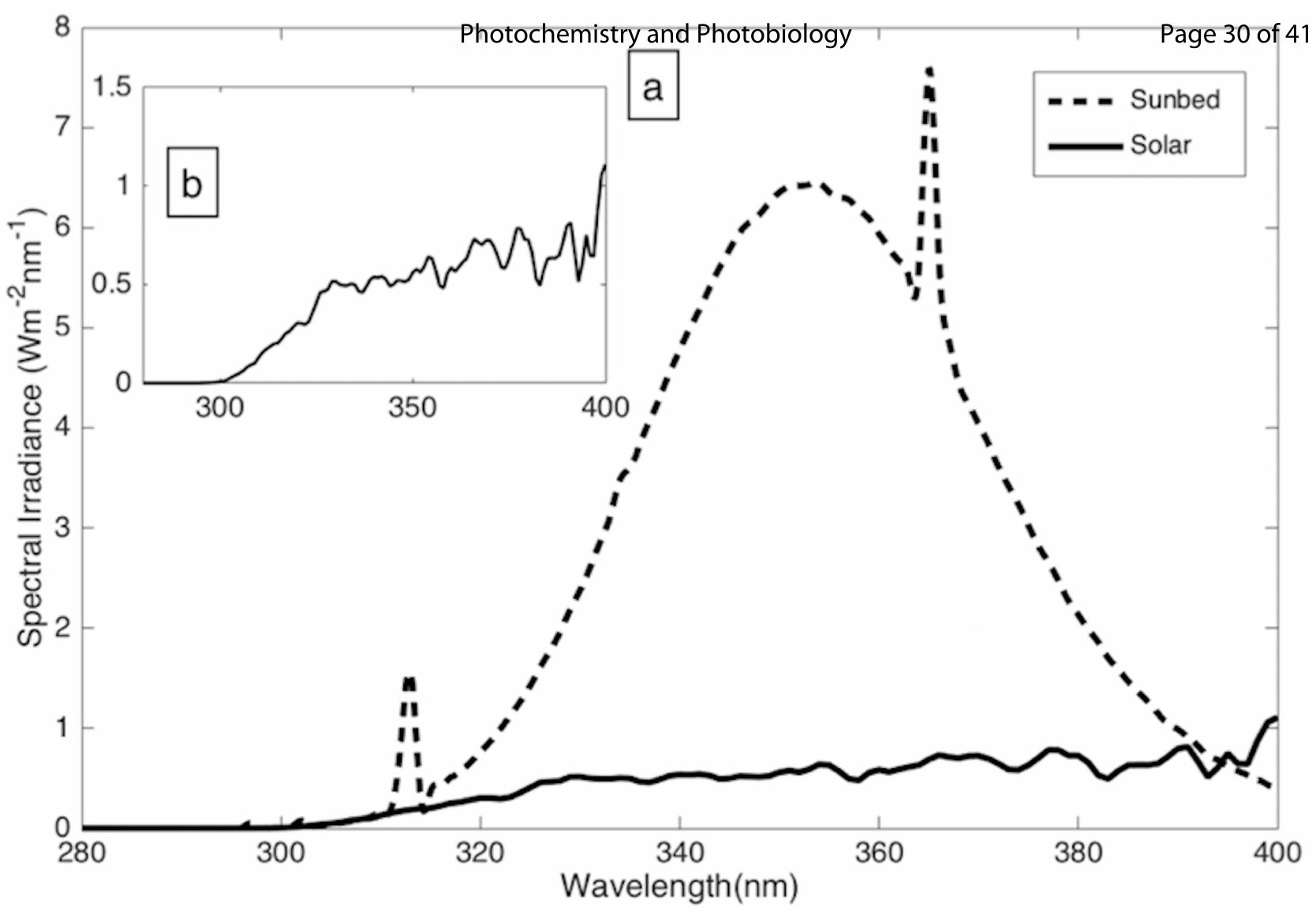




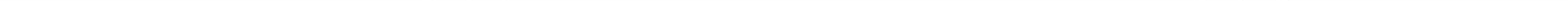


Page 333sof|âr (Type I)

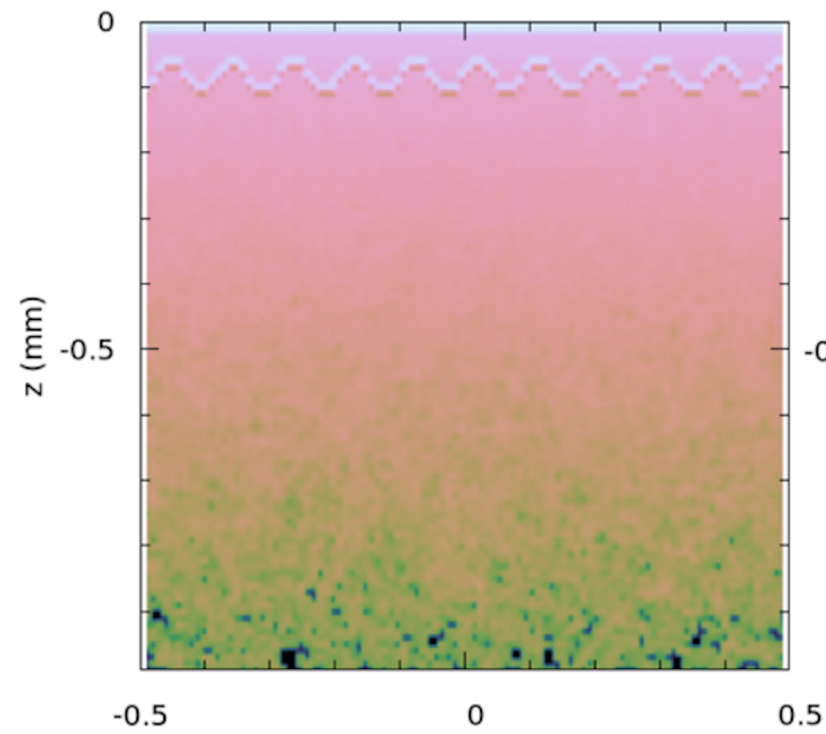

a) Solar (Type II)

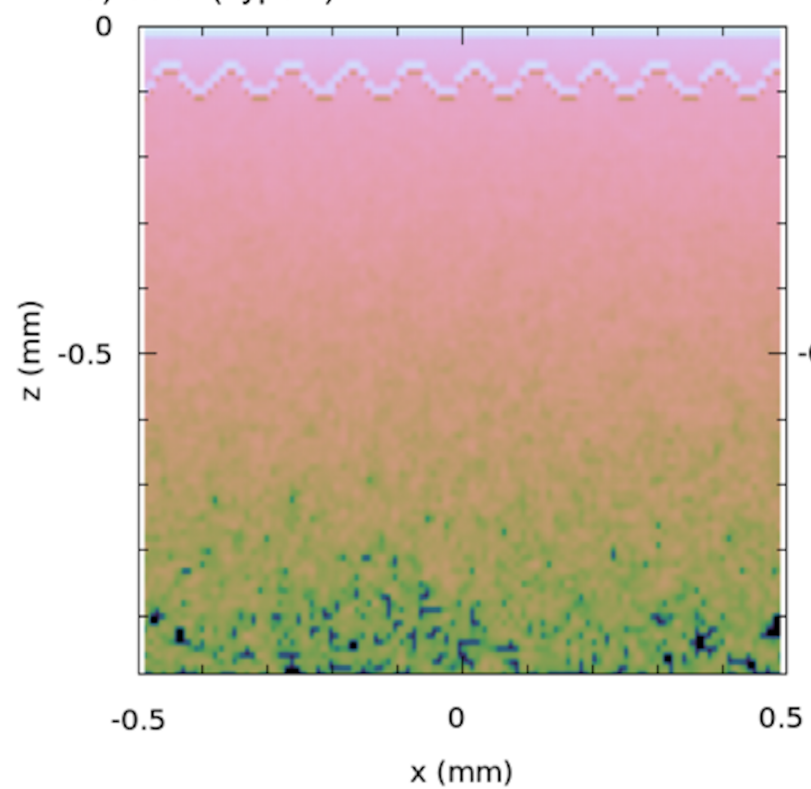

0

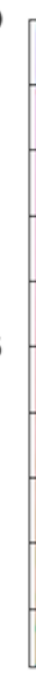

obglajighoydd (Type I)

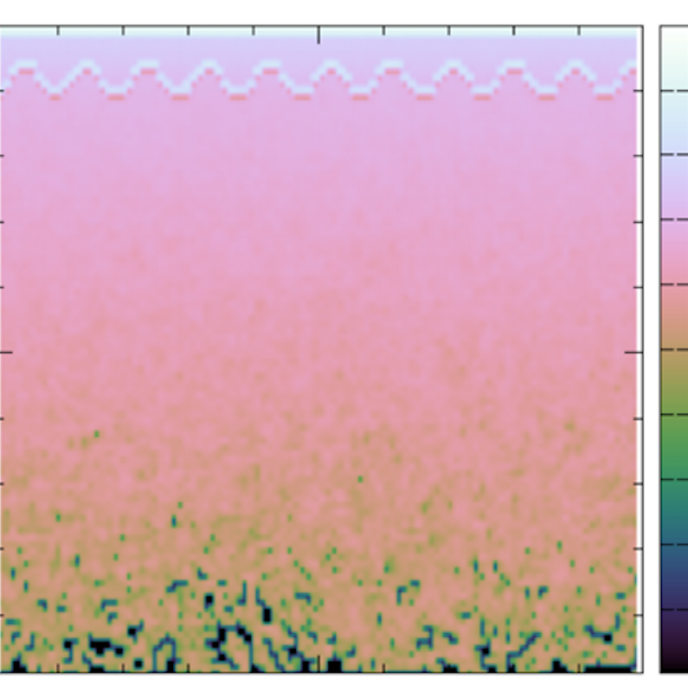

$-0.5$

b) Sunbed (Type II)

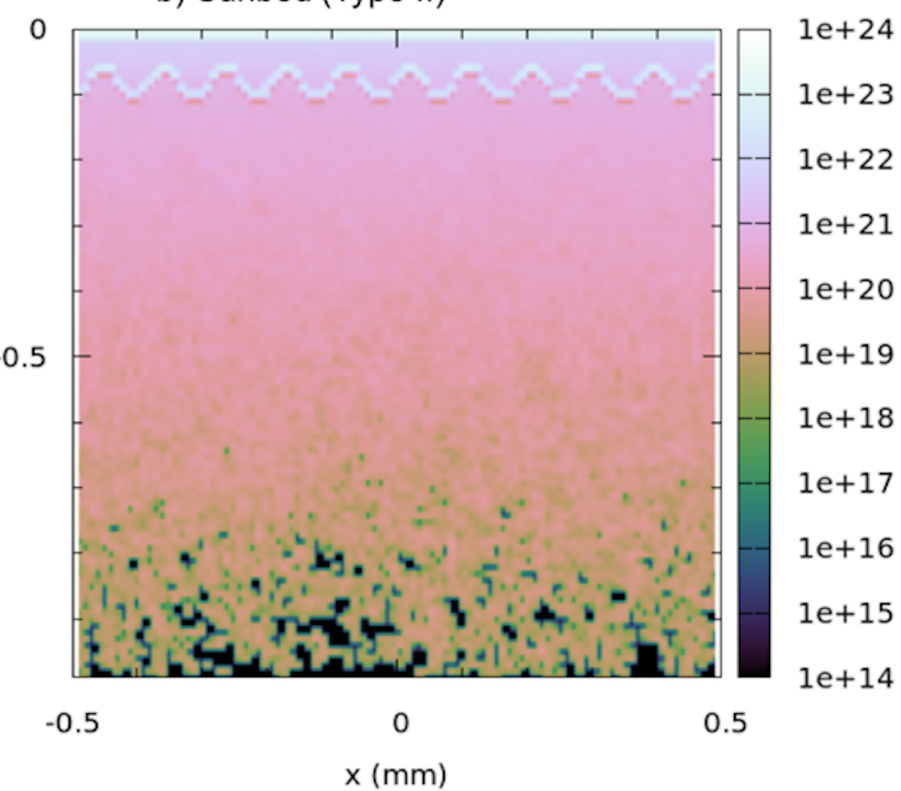

$1 e+24$

$1 e+23$

$1 e+22$

$1 e+21$

$1 e+20$

$1 e+19$

$1 e+18$

$1 e+17$

$1 e+16$

$1 e+15$

$1 \mathrm{e}+14$

0.5

Photons. $\mathrm{s}^{-1} \cdot \mathrm{cm}^{3}$ $+24$ $1 e+23$ $e+22$ $1 e+21$ $1 e+20$ $1 e+19$ $1 e+18$ $1 e+17$ $1 \mathrm{e}+16$ $1 e+15$ $1 e+14$ 
